\title{
AVALIAÇÃO DOS ASPECTOS ERGONÔMICOS DE FOOD TRUCKS LOCALIZADOS NO MUNICÍPIO DE SÃO PAULO
}

Rita de Cassia de Souza FERNANDES ${ }^{1}$

Marina de Almeida LIMA ${ }^{2}$

Noeli Aparecida Rosa de MORAIS ${ }^{3}$

Aline Cafer NICOLINI ${ }^{4}$

Mônica Glória Neumann SPINELLI ${ }^{5}$

\begin{abstract}
${ }^{1}$ Graduanda do curso de Nutrição do Centro de Ciências Biológicas e da Saúde da Universidade Presbiteriana Mackenzie. E-mail: ritadecsfernandes@gmail.com

${ }^{2}$ Graduanda do curso de Nutrição do Centro de Ciências Biológicas e da Saúde da Universidade Presbiteriana Mackenzie. E-mail: manutrimack@gmail.com

${ }^{3}$ Graduanda do curso de Nutrição do Centro de Ciências Biológicas e da Saúde da Universidade Presbiteriana Mackenzie. E-mail: noeli.rosa@gmail.com

${ }^{4}$ Graduanda do curso de Nutrição do Centro de Ciências Biológicas e da Saúde da Universidade Presbiteriana Mackenzie. E-mail: alinenicolini@ hotmail.com

${ }^{5}$ Nutricionista, mestre e doutora em Saúde Pública (Área de concentração: Nutrição) pela Faculdade de Saúde Pública da Universidade de São Paulo (USP), professora adjunta do curso de nutrição da Universidade Presbiteriana Mackenzie. E-mail: mônica.spinelli@ mackenzie.br
\end{abstract}

Recebido em: 28/12/2016 - Aprovado em: 05/05/2017 - Disponibilizado em: 01/07/2017

\begin{abstract}
RESUMO
Em um cenário de aumento da oferta de food trucks, esta pesquisa refere-se ao ambiente e às condições de trabalho dos funcionários deste setor. O objetivo foi avaliar ergonomicamente os aspectos ambientais e físicos de food trucks, bem como o estado nutricional e as condições de trabalho dos colaboradores. Os dados foram colhidos no último trimestre de 2016 durante o período vespertino em food trucks localizados no município de São Paulo. Utilizou-se a Análise Ergonômica do Trabalho (AET), aplicativos para determinar a luminosidade e os ruídos e um questionário adaptado onde também foi inserido o diagrama de áreas dolorosas e peso e alturas aferidos para cálculo de Índice de Massa Corporal (IMC). Todos os 17 food trucks analisados apresentaram níveis de luminosidade e ruído satisfatórios, apesar de a percepção dos colaboradores indicar o contrário. A temperatura foi classificada por 63,3\% como razoável ou ruim. Notou-se que $84 \%$ consideram fatores específicos nos food trucks que interferem no desempenho laboral, como o ruído e o layout dos veículos. Dos 30 colaboradores, 63,3\% pertencem ao gênero masculino e 63,3\% são eutróficos. Em adição, 96,7\% desempenhar dupla função com jornadas de trabalho variadas, o que impacta em cansaço (79,3\%) e em dor em alguma parte do corpo $(55,2 \%)$, sendo as mais sentidas nas pernas $(60 \%)$ e costas $(49,9 \%)$. Portanto, apesar de satisfatórias, as condições ergonômicas devem ser avaliadas para que não haja redução do desempenho, da produtividade e da qualidade de vida do trabalhador.
\end{abstract}

Palavras-chave: Carga de trabalho. Ergonomia. Estado nutricional. Food trucks. Funcionários.

\section{EVALUATION OF THE ERGONOMIC ASPECTS OF FOOD TRUCKS IN SÃO PAULO COUNTY}

\footnotetext{
ABSTRACT

In a scenario of increasing food truck offering, this research refers to the environment and working conditions of the employees of this sector. The objective was to evaluate ergonomically the environmental and physical aspects of food 
trucks, as well as the nutritional status and the working conditions of the collaborators. The data was collected in the last quarter of 2016 during the evening period in food trucks located in the city of São Paulo. We used the Ergonomic Work Analysis (EWA), applications to determine brightness and noise and an adapted questionnaire where we also inserted the diagram of week areas and weight and heights measured for calculation of Body Mass Index (BMI). All 17 food trucks analyzed showed satisfactory levels of luminosity and noise, although the perception of employees indicates otherwise. Temperature perception was classified by $63.3 \%$ as reasonable or poor. It was noted that $84 \%$ consider specific factors in food trucks that interfere with work performance, such as noise and vehicle layout. Of the 30 employees, $63.3 \%$ belong to the male gender and $63.3 \%$ are eutrophic. In addition $96.7 \%$ play dual role with varied work days, what impacts on fatigue (79.3\%) and in pain in any part of the body $(55.2 \%)$, being the deepest in the legs $(60 \%)$ and back $(49.9 \%)$. Therefore, despite of presenting satisfactory, ergonomic conditions should be evaluated so there is no reduction in performance, productivity and quality of life of the worker.

Keywords: Workload. Human engineering. Nutritional status. Food services. Manpower.

\section{INTRODUÇÃO}

Determinada por razões biológicas e questões sociais e culturais, a alimentação é uma das atividades mais importantes para o ser humano por suprir suas necessidades nutricionais e sensoriais, englobando desde a produção à transformação e disponibilidade de refeições aos indivíduos (BRASIL, 2014; ALEVATO; ARAÚJO, 2009; BRASIL, 2006).

Em virtude das intensas mudanças sociais ocorridas nas últimas décadas, caracterizados pelo desenvolvimento industrial e jornada de trabalho extensa com maior participação da mulher, o setor de alimentação coletiva vem se tornando um mercado cada vez mais representativo (AKUTSU et al., 2005; HENRIQUES et al., 2014; LOURENÇO, MENEZES, 2008). O número de refeições realizadas fora de casa, além de já surpreender em países desenvolvidos, representa no Brasil 33,1\% dos gastos totais com alimentação (IBGE, 2010).

De acordo com Abreu, Spinelli e Souza Pinto (2016), a alimentação coletiva é representada pelas atividades de alimentação e nutrição, sendo estas realizadas em Unidades de Alimentação e Nutrição (UAN), responsáveis por promover um serviço de fornecimento de refeições balanceadas dentro dos padrões dietéticos e higiênicos, de modo a atender as necessidades nutricionais de seus clientes. Um dos tipos mais comuns devido ao estilo de vida contemporâneos são as UAN comerciais.

Além de restaurantes fixos, devido à rapidez do serviço no preparo dos alimentos, atualmente o mercado de comida de rua em vias públicas vem obtendo destaque por estar próximo a locais de trabalho e estudo de grande parte da população, tendo forte adesão por não necessitar de etapas adicionais de processamento, permitindo consumo imediato ou posterior (BEZERRA; MANCUSO; HEITZ, 2014; WHO, 1996).

Assim, de acordo com Lippel (2002), com o objetivo de atender às necessidades dos diversos tipos de clientes, bem como devido à superação de crises ou recessões econômicas, inovações na área de alimentação coletiva tendem a surgir com serviços diferenciados 
no atendimento, preço do produto, qualidade das instalações e tipos de serviço. Nessa perspectiva, em 2008 chefes de cozinha americanos famosos adotaram uma nova estratégia de venda: os food trucks, restaurantes itinerantes montados em veículos automotores adaptados para o serviço, preparo e venda de alimentos, que, por possuírem custos operacionais inferiores, tornaram-se referência mundial e conquistaram parte significativa dos consumidores (BOA NOVA, 2014; ROSS et al., 2015).

Por se destacarem quanto à qualidade, valor e velocidade, no Brasil os food trucks fizeram sucesso em relação aos demais restaurantes de refeições rápidas (ROOS et al., 2015). Segundo Kregor (2015), essa não foi uma inovação radical, mas sim uma inovação radical que mudou uma indústria, tornando-se parte do cotidiano de muitas pessoas.

Carvalho et al. (2007) ressalta que, para a produção de refeições, alguns fatores estão diretamente envolvidos, como o número de operadores, as técnicas de preparo, a infraestrutura e o tipo de alimento utilizado e comercializado. Mas, atualmente, os funcionários de UAN estão cada vez mais submetidos à alta produtividade em tempo limitado, devido à demanda crescente de pedidos, sendo que preparam os alimentos, na maioria das vezes, em cozinhas cujas condições se apresentam ergonomicamente inadequadas para o trabalho (ABREU; SPINELLI; SOUZA PINTO, 2016).

Apesar do desenvolvimento tecnológico, ainda é possível observar, no setor de alimentação coletiva, a existência de ambientes de trabalho com equipamentos inadequados, ruídos em excesso, assim como calor, umidade, ventilação e iluminação insatisfatórios, que podem levar o trabalhador ao desgaste físico e psicológico (CONCEIÇÃO; CAVALCANTI, 2001).

O colaborador de uma UAN é exposto ao cansaço físico de diversas formas: movimentação manual intensa e repetitiva; postura inadequada devido à quantidade de tempo que os funcionários ficam na posição em pé; uso de força excessiva; jornada de trabalho prolongada, dentre outros. Como consequências desses esforços, Abreu, Spinelli e Souza Pinto (2016) destacam quedas de produtividade, problemas de saúde e até mesmo acidentes de trabalho. Para evitálos, é necessário não somente promover o uso de Equipamentos de Proteção Individual (EPI), como também oferecer a esse colaborador condições seguras e saudáveis que promovam um desempenho adequado de modo a se evitarem dores; cansaço; insatisfação; estresse; edemas e varizes; aparecimento de lesões; agravos ao sistema musculoesquelético, além de problemas nos membros superiores e inferiores, região lombar e coluna vertebral (FERNANDES, 
2014; SIQUEIRA et al., 2011; LOURENÇO; MENEZES, 2008; SILVA et al., 2008).

De tal forma, atualmente, essa relação entre a exposição do corpo humano aos riscos presentes no seu ambiente de trabalho é evidenciada pelo aumento do desenvolvimento de doenças ocupacionais, como os Distúrbios Osteomusculares Relacionados ao Trabalho (DORT) e as Lesões por Esforços Repetitivos (LER) (MESQUITA et al., 2004).

É neste cenário produtivo que surge a aplicação da ergonomia em UAN. A ergonomia, definida como a ciência que estuda a relação entre o homem e seu trabalho, visa adaptar esse ambiente após analisar a interação do corpo humano com a carga física a qual é exposto. Perante algumas finalidades básicas, também busca melhorar a conservação da saúde dos trabalhadores ao mesmo tempo em que promove o funcionamento satisfatório do sistema técnico do ponto de vista da produção e segurança, além de seu bem-estar, resultados e eficiência satisfatórios (CARVALHO, 2005; FERNANDES, 2014). Lida (2005) também refere que a ergonomia física busca reduzir as exigências biomecânicas dos trabalhadores, readequando, caso haja a necessidade, seus postos de trabalho ou os equipamentos utilizados, como máquinas e utensílios.

Desconsiderando-se o pré-preparo dos alimentos, feito em cozinhas de apoio, ao ser realizada uma análise em relação aos food trucks, muitas particularidades são observadas, na produção e finalização dos alimentos feitos nas vias públicas, principalmente o que se refere à estrutura física, que possui como características uma cozinha montada em um veículo adaptado para essa finalidade, sendo grande a possibilidade de inadequação no que se refere às questões ergonômicas.

Torres et al. (2006) destacam o layout dos estabelecimentos como elemento determinante na utilização do espaço e do conforto do usuário, visto que o arranjo físico além de dispor racionalmente o processo produtivo, também se preocupa com áreas de movimentação, alcances, manuseios de pesos e disposição de mobiliário. Não é possível observar, de tal forma, nos food trucks um fluxo de produção ordenado e sem cruzamentos, o que pode impactar nas condições de trabalho.

Ademais, por estar inserido rotineiramente em grandes cidades e devido à busca pelos comerciantes por pontos com grande fluxo de pessoas, como avenidas, que também contam com maior concentração de veículos, visando uma maior comercialização, os food trucks inseridos nesses locais podem expor o colaborador a ruídos adicionais aos habituais de cozinhas, podendo prejudicar sua audição e seu desempenho.

Outra característica importante refere-se à sua mão-de-obra, normalmente composta por poucos funcionários devido ao espaço 
diminuto, o que poderia levar a sobrecarga no serviço. De certa forma pode até diferenciarse de restaurantes fixos, que possuem diversas áreas de trabalho e monotonia de função; mas, devido à escassez de colaboradores, maior pode ser a sobrecarga imposta a eles, além da permanência de períodos prolongados na postura em pé (COLARES; FREITAS, 2007).

Orofino (2004) ressalta que a realização das atividades em pé, sem apoio e sem a adequação do meio, impõe aos funcionários uma postura forçada, principalmente no que se refere à higienização de equipamentos, utensílios e instalações. Por exigir um trabalho estático da musculatura envolvida, tal condição pode provocar uma fadiga muscular e um aumento importante da pressão hidrostática do sangue nas veias das pernas, levando ao desenvolvimento de transtornos circulatórios, como os já citados (JORGE, 2003).

Em relação à iluminação, apesar de em grande parte do dia os food trucks se utilizarem de fonte de luz natural, é necessário avaliar se a luminosidade atende aos parâmetros das normas regulamentadoras, de modo que promova boa visibilidade aos funcionários e permita adequada manipulação de alimentos. Quando utilizada fontes de iluminação artificial, devem adequar-se às normas sanitárias (ABREU; SPINELLI; SOUZA PINTO, 2016).

Outro fator que poderia impactar diretamente o colaborador se refere ao recebimento de mercadorias, feito muitas vezes de maneira inadequada, sem o auxílio de equipamentos, uma vez que é característico desse nicho de mercado que os ingredientes ou produtos pré-preparados sejam recebidos pelos próprios funcionários após o transporte realizado inadequadamente em carros de passeio.

Além do ambiente de trabalho em si, também é importante, para manutenção do desempenho e da saúde, que os colaboradores das UAN possuam um estado nutricional adequado, ou seja, que se mantenham eutróficos (LOURENÇO et al., 2006). Tal condição pode possuir relação direta com o ambiente de trabalho, mas, principalmente, com as escolhas e preferências dos funcionários, cuja alimentação vem sendo impactada diretamente pela industrialização desenfreada que colocou à disposição da população produtos ultraprocessados ricos em sódio, gordura saturada e açúcar (TRAEBERT et al., 2004). Ademais, propagandas midiáticas e a rotina de trabalho intensa também podem interferir na introdução e manutenção desses alimentos na dieta dos colaboradores, bem como a exposição destes aos próprios alimentos que auxiliam no preparo para a venda nos food trucks, cujo cardápio, para maior aceitação ou devido ao tempo de preparo menor, pode ser semelhante ao comercializado em redes de fast foods (MATOS; PROENÇA, 2003) 
Um possível excesso de peso pode contribuir para tornar a atividade mais desgastante e cansativa, gerando uma sobrecarga à coluna vertebral e impactando diretamente no seu desempenho, bem como levar ao desenvolvimento de doenças crônicas não transmissíveis (DCNT), como hipertensão arterial e diabetes, diminuindo sua qualidade de vida (LOURENÇO et al., 2006; MATOS; PROENÇA, 2003).

De tal forma, por ser um setor emergente, é necessário avaliar se o ambiente físico dos food trucks está de acordo com os aspectos ergonômicos desejados, bem como se os funcionários se encontram com a saúde física e mental em condições adequadas para continuar desempenhando um serviço de qualidade, mantendo o rendimento adequado do estabelecimento sem que prejuízos à sua saúde sejam identificados ou surjam futuramente, de forma a se reduzir erros, aumentando a produtividade e evitar absenteísmo ou afastamentos por motivos de doenças ocupacionais.

Assim sendo, o objetivo deste trabalho foi avaliar o ambiente de trabalho de food trucks relacionando a ergonomia com seus aspectos ambientais e físicos no que se referem à luminosidade, ruídos e percepção de temperatura, bem como estado nutricional dos funcionários, repetição de atividades, carregamento de peso em excesso e as partes do corpo mais exigidas durante a atividade laboral.

\section{METODOLOGIA}

O estudo foi observacional descritivo de delineamento transversal realizado a partir de coleta de dados primários em food trucks localizados no município de São Paulo. A população do estudo foi composta por funcionários de food trucks, sendo utilizada, em ambos os casos, a amostragem por conveniência. O período de coleta foi entre os meses de outubro e novembro de 2016, no período vespertino e contou com a participação de 30 funcionários.

Utilizou-se a Análise Ergonômica do Trabalho (AET), que possui caráter qualitativo e quantitativo, para verificar os aspectos ambientes e físicos, além da verificação de luminosidade e ruídos do ambiente (MATOS; PROENÇA, 2003). O nível de luminosidade (iluminância) foi medido por meio do aplicativo "Medidor de luz 02 Led", sendo a média dos dados obtidos pelo aplicativo apresentada em LUX; os ruídos, pelo aplicativo "Decibelímetro", que apresentou o nível de pressão sonora em decibéis (dB). Foram considerados os parâmetros para luminosidade de 250 LUX (SILVA JUNIOR, 2005) e, quanto aos ruídos, o nível máximo de $85 \mathrm{~dB}$ para uma jornada de oito horas (BRASIL, 1978).

Para verificar o uso de equipamentos de proteção; carregamento de peso; atividades repetitivas; percepção de temperatura e outras informações relevantes ao tema, utilizou-se um questionário adaptado de Maciel et al. 
(2013), sendo feito um levantamento de áreas do corpo mais exigidas durante a atividade laboral por meio de um diagrama de áreas dolorosas.

Foi calculado o Índice de Massa Corporal (IMC) para determinar o estado nutricional dos colaboradores, dividindo-se o peso $(\mathrm{kg})$ pela altura ao quadrado $\left(\mathrm{m}^{2}\right)$, referidos pelos sujeitos de pesquisa. A classificação do estado nutricional se baseou nos pontos de corte propostos pela Organização Mundial da Saúde (WHO, 1995: WHO, 1997), sendo baixo peso (IMC < 18,5 $\mathrm{kg} / \mathrm{m}^{2}$ ), normal ou eutrofia (IMC entre 18,5 e $24,9 \mathrm{~kg} / \mathrm{m}^{2}$ ), sobrepeso (IMC entre 25 e 29,9 $\mathrm{kg} / \mathrm{m}^{2}$ ) e obesidade (IMC $\geq 30 \mathrm{~kg} / \mathrm{m}^{2}$ ).

Os dados foram analisados por meio de estatística descritiva, com apresentação de gráficos e porcentagens dos dados analisados, além de comparados com a literatura. Foi utilizado o coeficiente de correlação de Pearson para ver se há relação entre a dor sentida e o tempo de trabalho atual.

Foram devidamente explicados aos donos ou responsáveis pelo estabelecimento, bem como aos funcionários que participaram como sujeitos de pesquisa, os objetivos e procedimentos do estudo e os riscos, mesmo que mínimos. Ao consentirem voluntariamente em participar, assinaram o termo de consentimento livre e esclarecido (TCLE). Os procedimentos para o desenvolvimento deste estudo respeitaram as diretrizes e normas que regulamentam as pesquisas envolvendo humanos, aprovadas pela resolução $\mathrm{n}^{\circ} 510$ (BRASIL, 2016). No banco de dados da pesquisa principal foram mantidos o anonimato e a confidencialidade dos dados. Esse projeto foi aprovado pelo Comitê de Ética em Pesquisa sob o número CAAE - 48483015.7.0000.0084.

\section{RESULTADOS E DISCUSSÃO}

Foi avaliado o ambiente de trabalho de 17 food trucks localizados em 5 food parks do município de São Paulo.

A média encontrada de luminosidade foi de 1730,9 \pm 1281,6 LUX, sendo que apenas dois estabelecimentos $(11,8 \%)$ estavam abaixo dos parâmetros propostos por Silva Junior (2005). Porém, foram observados resultados exorbitantes que excederam os esperados, o que se configura como certa limitação no presente estudo, visto que os food trucks se encontram em áreas ao ar livre e, tendo a coleta de dados sido realizada no período vespertino, mesmo com a presença de toldos em alguns estabelecimentos, houve forte influência de iluminação natural e suas variações. Não se pode deixar de ressaltar, porém que, estando acima dos parâmetros, a garantia de um conforto visual é importante para se manter a eficiência do trabalho e se reduzirem doenças visuais, bem como possíveis cefaléias, irritação e lacrimejamento (FONSECA, 2009). 
Em relação aos níveis de ruídos, que a princípio se configuravam como uma preocupação devido ao fato de os food trucks se localizarem em vias públicas, foram encontrados resultados satisfatórios: a média foi de $66,7 \mathrm{~dB}$, sendo o desvio padrão de 5,7 $\mathrm{dB}$, estando dentro do máximo permitido (BRASIL, 1978). De tal forma, o ambiente propicia uma facilitação na comunicação dos colaboradores e evitará possíveis alterações de humor, perda de concentração e da capacidade auditiva, sintomas característicos de ambientes com altos ruídos (ALBUQUERQUE et al., 2012). Entretanto, por não terem sido analisados em um período de grande fluxo de clientes e atendimentos, esses estabelecimentos ainda podem ter a performance dos funcionários prejudicada, visto que maior número de pessoas contribui de maneira significativa para o aumento dos níveis de ruído.

No gráfico 1, observa-se a percepção dos colaboradores frente aos dois itens analisados. Apesar da alta luminosidade encontrada por meio do uso do aplicativo, $63,3 \%$ dos funcionários referiram bom conforto visual no posto de trabalho. Quanto aos níveis de ruído, $70 \%$ dos colaboradores classificaram como sendo ruim ou razoável, mesmo os parâmetros estando dentro do estabelecido (BRASIL, 1978).
Gráfico 1 - Percepção de conforto visual e níveis de ruído de colaboradores de food trucks localizados no município de São Paulo, 2016.

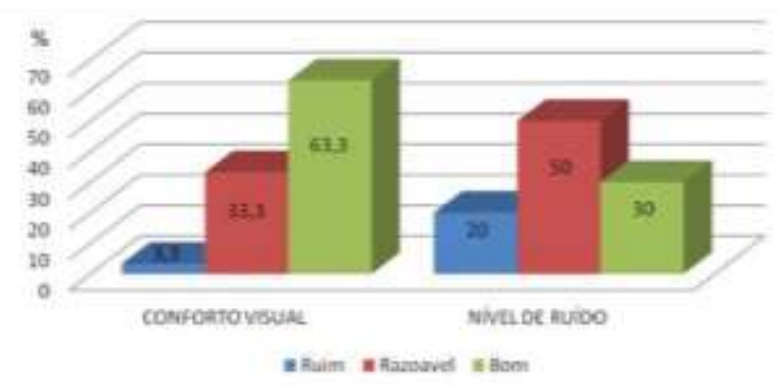

A percepção da temperatura do ambiente foi relatada como boa por apenas $36,7 \%$, enquanto que $40 \%$ relataram como razoável e 23,3\% como ruim. Sabe-se que dentro do ambiente de trabalho dos food trucks podem ser sentidas muitas oscilações de temperatura, tanto por razões ambientais (vento ou calor intenso), como devido à manipulação e preparação dos alimentos aos consumidores. De tal forma, ressalta-se que se garantido o conforto térmico, uma contribuição positiva se dará em relação ao desempenho (ALBUQUERQUE et al., 2012).

Também foi analisado se o ambiente de trabalho dos food trucks agia de maneira diferente sobre o trabalho dos funcionários, sendo perguntado a estes se anteriormente haviam trabalhado em cozinhas ou restaurantes comerciais fixos, o que foi relatado por $83,3 \%(n=25)$ dos colaboradores. Destes, 84,0\% ( $\mathrm{n}=21)$ acreditam que sim, sendo que os fatores que mais interferem no desempenho, tanto positivamente quanto negativamente, são apresentados no Gráfico 2 : 
Gráfico 2 - Fatores que interferem no desempenho de funcionários de food trucks em relação a cozinhas fixas, São Paulo, 2016.

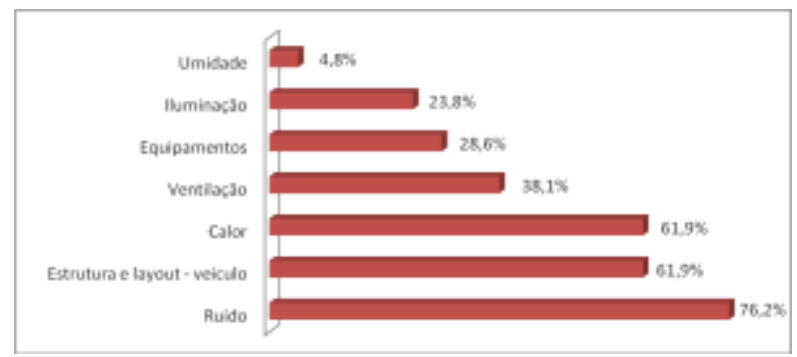

Quanto à análise e avaliação dos colaboradores, dos 30 funcionários participantes do estudo, 63,3\% eram pertencentes ao gênero masculino, com uma média de idade de 28,6 \pm 5,1 anos.

A média de IMC do grupo foi de $25,1 \pm$ $3,6 \mathrm{~kg} / \mathrm{m}^{2}$, estando às classificações quanto ao estado nutricional descritas no Gráfico 3. Diferentemente do cenário epidêmico de sobrepeso e obesidade no Brasil, os participantes encontram-se com percentuais inferiores aos encontrados pelo Ministério da Saúde (BRASIL, 2014), visto que 23,3\% estão com sobrepeso e 13,3\% com obesidade grau 1. Comparando-se a estudos com colaboradores de UAN, Lourenço et al. (2006) encontrou um maior percentual de sobrepeso $(39,1 \%)$ e obesidade grau 1 (15,2\%), além de indivíduos com obesidade grau $2(13,0 \%)$, o que não foi encontrado no presente estudo. Apesar disso, é válido reforçar que o excesso de peso pode prejudicar a qualidade de vida do trabalhador por ser fator de risco para DCNT e tornar as atividades laborais mais exaustivas (BRASIL, 2014; MATOS; PROENÇA, 2003)
Gráfico 3 - Classificação de estado nutricional de colaboradores de food trucks localizados no município de São Paulo, 2016.

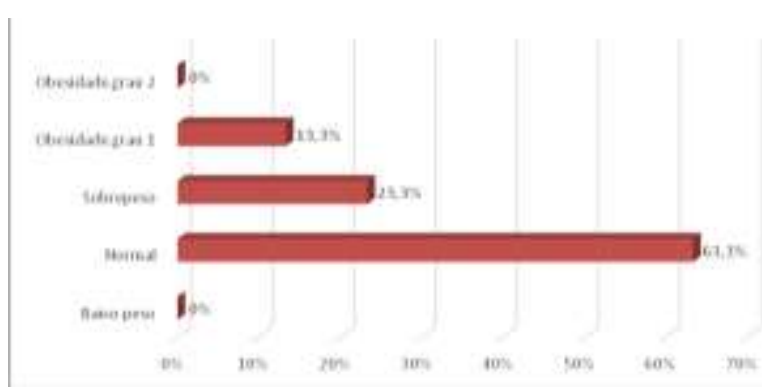

Dos colaboradores analisados, 76,7\% receberam treinamento antes de iniciar no serviço e apenas $13,3 \%$ sentem que houve alguma defasagem para um melhor desempenho. Uma particularidade encontrada refere que $96,7 \%$ dos funcionários desempenham dupla função no food truck, sendo estas de atendente e manipulador de alimentos, o que pode ser justificado pelo espaço diminuto encontrado. Além disso, as jornadas de trabalho diárias relatadas de seis horas (3\%), oito horas (60\%), doze horas $(30 \%)$ e quinze horas $(3 \%)$ podem levar a um esforço físico muito intenso pelos funcionários, visto que a demanda desses estabelecimentos acompanha o ritmo do estilo de vida cotidiano, cuja produção deve ser feita em tempo limitado e sem a perda de qualidade.

As atividades mais referidas como repetitivas referem-se à reposição de mercadorias $(26,6 \%)$, montagem de lanches (26,6\%), lavagem de louça (16,7\%), atendimento e caixa $(16,7 \%)$ e corte de alimentos (13,3\%) que, de acordo com Dourado e Lima (2011), apresenta-se como 
um erro ergonômico que pode levar a doenças ocupacionais.

Metade dos colaboradores não costuma carregar peso durante nenhuma atividade desenvolvida no food truck, e, dos que carregam, apenas 13,3\% consideram o peso maior do que pode carregar. Tais resultados, apesar da porcentagem encontrada, podem ser considerados positivos por Lourenço et al. (2006) por não acarretar aos funcionários dores na coluna e má postura advindas dessa atividade.

Em relação aos desconfortos sentidos pelos funcionários, os mais referenciados foram o cansaço $(79,3 \%)$ e a dor em alguma parte do corpo (55,2\%). Ademais, $24,1 \%$ relataram sentir estalos; $20,7 \%$ sensação de peso; $\quad 17,2 \%$ dolorimento; $10,3 \%$ formigamento; $6,9 \%$ perda de força e $3,4 \%$ limitação do movimento. Destaca-se, no Gráfico 4, a intensidade desses desconfortos mais recorrentes:

Gráfico 4 - Intensidade de desconfortos sentidos por colaboradores de food trucks em localizados no município de São Paulo, 2016.

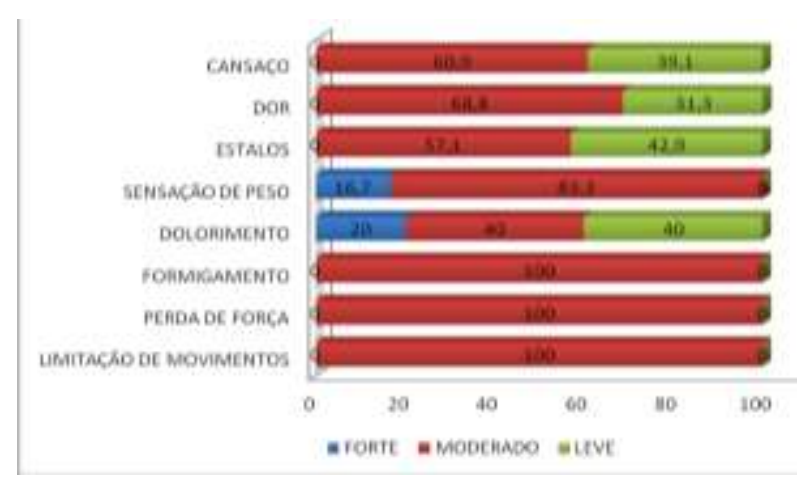

Relacionando-se a sensação de peso relatada e o estado nutricional, observou-se que $16,7 \%$ dos colaboradores encontram-se com sobrepeso e $66,7 \%$ com obesidade grau I, o que corrobora os estudos de Matos e Proença (2003), visto que esse desconforto musculoesquelético advém da maior sobrecarga empregada na coluna vertebral.

Entre as partes do corpo onde mais foram relatados os desconfortos estão: pernas $(60,0 \%)$, costas $(49,9 \%)$, tornozelos $(26,6 \%)$, ombros $(23,3 \%)$, punhos $(16,6 \%)$ e braço $(13,3 \%)$. Os colaboradores referiram que as pernas e as costas são as áreas que mais demandam esforço na realização de atividades, sendo responsáveis por $43,3 \%$ e $30,0 \%$ respectivamente. Entretanto, 33,3\% não fizeram menção a nenhuma área do corpo.

É válido ressaltar que $24,1 \%$ dos colaboradores não acreditam que tais desconfortos aumentam durante a jornada de trabalho, mas $65,5 \%$ relataram tal ocorrência, assim como nas horas extras $(10,3 \%)$ e no período noturno $(6,9 \%)$, o que tende a prejudicar a performance e pode levar ao desenvolvimento de doenças ocupacionais. Porém, independentemente de aumentar ou não, $83,3 \%$ acreditam que o que sentem e referiram está relacionada ao serviço, tendo tempo de dor sentida uma forte correlação $(p=$ 0,73) com o período de trabalho no setor atual. Todos os funcionários acreditam que há uma melhoria do desconforto durante o repouso à noite ou nos finais de semana $(6,9 \%)$. 
Quando perguntado se há algum intervalo durante o expediente, observou-se uma peculiaridade: como os alimentos só são preparados sob a demanda do consumidor e os food trucks analisados estavam inseridos em food parks, 23,3\% afirmaram que enquanto aguardam algum cliente, realizam uma pausa superior à uma hora, além do horário de almoço (FERNANDES et al., 2016).

Em relação ao uso de equipamentos de Equipamentos de Proteção Individual (EPI), $13,3 \%$ referiram nunca utilizar e 43,3\% sempre a luva e a touca, o que poderia levar a uma contaminação cruzada ou a acidentes de trabalho caso não for trocada quando houver mudança de atividade ou retirada durante procedimentos que envolvam o calor (SÃO PAULO, 2011). Além disso, apenas 16,7\% referiram utilizar sapato fechado e, quanto à uniformização, foi encontrada uma total não conformidade, equiparando-se aos estudos de Fernandes et al. (2016), o que poderia comprometer a integridade física do trabalhador, visto que não se encontravam fechados, completos e apropriados para a atividade, conforme Portaria 2619 (SÃO PAULO, 2011).

\section{CONCLUSÃO}

A avaliação do ambiente de trabalho dos food trucks em relação à ergonomia e seus aspectos ambientais e fixos revelou condições satisfatórias, tanto em relação à luminosidade, quanto ao ruído, apesar de a percepção dos colaboradores não se equiparar aos resultados obtidos, além de $63,3 \%$ relatarem a sensação térmica como razoável ou ruim, fatores estes que podem impactar na performance do funcionário. Porém, as condições de trabalho as quais são submetidas o trabalhador, como dupla função e atividades repetitivas, refletem em cansaço e dor com intensidade moderada em partes variadas do corpo, sendo relatadas como mais exigidas nas atividades as pernas e as costas, devido a um esforço físico exacerbado,

Perceber a importância da ergonomia em uma unidade de alimentação e nutrição configura-se como essencial para evitar diminuição de produtividade e de riscos de doenças ocupacionais, sendo, para isso, necessário atentar-se não só no que se refere ao ambiente e à qualificação e trabalho dos funcionários, como também em relação ao estado nutricional dos mesmos.

\section{REFERÊNCIAS}

ABREU, E.S.; SPINELLI, M.G.N.; SOUZA PINTO, A.M. Gestão de Unidades de Alimentação e Nutrição: um modo de fazer. Ed. Metha, $6^{\text {a }}$ ed. São Paulo, 2016, $392 p$.

AKUTSU, R.C. et al. Adequação das boas práticas de fabricação em serviços de alimentação. Rev. Nutr., v.18, n.13, p.419427, 2005. 
ALBUQUERQUE, E.N. et al.Riscos físicos em uma unidade de alimentação e nutrição: implicações na saúde do trabalhador. Rev.

Assoc. Bras. Nutr. v.4, n.5, 2012.

ALEVATO, H.; ARAÚJO, E.M.G. Gestão, organização e condições de trabalho. In: V CONGRESSO NACIONAL DE

EXCELÊNCIA EM GESTÃO: GESTÃO DO CONHECIMENTO PARA A

SUSTENTABILIDADE. Niterói, RJ.

Disponível em:

<http://www.inovarse.org/filebrowser/downlo ad/10036>. Acesso em: 03 ago. 2016.

BEZERRA, A.C.D.; MANCUSO, A.M.C.; HEITZ, S.J.J. Alimento de rua na agenda nacional de segurança alimentar e nutricional: um ensaio para a qualificação sanitária no Brasil. Ciência \& Saúde Coletiva, v.19, n.5, p.1489-1494, 2014.

BOANOVA, A.B. Análise e interpretação de denúncias sobre alimentos como ferramenta de gestão no município de São Paulo. Tese (Doutorado - Programa de PósGraduação em Saúde Pública. Área de concentração: Serviços de Saúde Pública) Faculdade de Saúde Pública da Universidade de São Paulo, São Paulo, 2014.

BRASIL. Diário Oficial da união (BR).

Resolução n⿳ 510, de 07 de abril de 2016. Disponível em:

<http://conselho.saude.gov.br/resolucoes/201 6/Reso510.pdf>. Acesso em: 10 jun. 2016.

BRASIL. Ministério da Saúde. Secretaria de Atenção à Saúde. Departamento de Atenção Básica. Guia alimentar para a população brasileira. 2 ed. Brasília: 2014.

BRASIL. Ministério da Saúde. Secretaria de Atenção à Saúde. Guia alimentar para a população brasileira: promovendo a alimentação saudável. 1 ed. Brasília: 2006.

BRASIL. Ministério do trabalho. NR 15 Atividades e operações Insalubres. 1978.
Disponível em:

$<$ http://trabalho.gov.br/images/Documentos/S

ST/NR/NR15/NR15-ANEXO15.pdf >. Acesso em: 05 set. 2016.

CARVALHO, A.A. Análise ergonômica do trabalho (AET) em uma cozinha industrial de restaurante self-service, Viçosa - MG. In: III WORKSHOP DE ANÁLISE ERGONÔMICA DO TRABALHO NA UFV; Minas Gerais; 2007. Disponível em: $<$ https://pt.scribd.com/doc/149686217/11analise-ergonomica-do-trabalho-aet-em-umacozinha-industrial-de-restaurante-self-servicevicos >.Acesso em: 05 set. 2016.

\section{CARVALHO, C.C.S. Diagnóstico do} ambiente, da segurança e da saúde do trabalhador na pós-colheita de café. Dissertação (Mestrado em Engenharia Agrícola). Universidade Federal de Lavras, 2005.

COLARES, L.G.T.; FREITAS, C.M.

Processo de trabalho e saúde de trabalhadores de uma unidade de alimentação e nutrição: entre a prescrição e o real do trabalho. Cad. Saúde Pública, Rio de Janeiro, v.23, n.12, p.3011-3020, 2007.

CONCEIÇÃO, M.L.; CAVALCANTI, C.L. Avaliação dos Equipamentos de Proteção Individual (EPIs) na Unidade de Alimentação e Nutrição (UAN) do Restaurante Universitário da UFPB. Rev. Conceitos. João Pessoa, v.4, n.5, p.105-108, 2001.

DOURADO, M.M.J.; LIMA, T.P. Ergonomia e sua importância para trabalhadores de unidades de alimentação e nutrição. Ensaio de ciência: ciências biológicas, agrárias e da saúde. v.15, n.4, p.183-196, 2011.

FERNANDES, C.A. Ergonomia e projeto: contribuições no projeto de ferramentas manuais para agricultura familiar com ênfase nos reflexos físicos da atividade de extração manual de mandioca. Tese (Mestrado - Programa de Pós-Graduação em 
Engenharia de Produção) - Universidade

Federal e Santa Catarina, Florianópolis, 2014.

FERNANDES, R.C.S. et al. Análise das condições higienicossanitárias de food trucks do município de São Paulo. In: XVII CONGRESSO INTERNACIONAL DE GASTRONOMIA E NUTRIÇÃO. São Paulo, 2016. Disponível em:

<http://www.nutricaoempauta.com.br/revistae letronica/67.phps>. Acesso em: 13 nov. 2016.

HENRIQUES, P. et al. Atitudes de usuários de restaurante "self-service": um risco a mais para a contaminação alimentar. Cad. Saúde Colet., Rio de Janeiro, v.2, n. 3, p. 266-74, 2014.

IBGE. Instituto de Geografia e Estatística. Pesquisa de Orçamentos Familiares 20082009. Jun. 2010. Disponível em: <http://www.ibge.gov.br/home/estatistica/pop ulacao/condicaodevida/pof/2008_2009/POFp ublicacao.pdf>. Acesso em: 14 mar. 2016.

JORGE, M.C.T.C. et al. A postura de trabalho em pé: um estudo com trabalhadores lojistas. Tese (Mestrado Programa de Pós-Graduação em Engenharia de Produção) - Universidade Federal e Santa Catarina, Florianópolis, 2003.

KREGOR, B. Food Trucks, Incremental Innovation, and Regulatory Ruts. Rev. Dialogue, v. 82, p. 1, 2015. Disponível em: $<$ http://chicagounbound.uchicago.edu/cgi/vie wcontent.cgi article $=11767 \&$ context $=$ journal _articles>. Acesso em: 09 set. 2016.

LIPPEL, I.L. Gestão de custos em restaurantes: utilização do método $\mathrm{ABC}$. Tese (Mestrado - Programa de Pós-Graduação em Engenharia de Produção) - Universidade Federal e Santa Catarina, Florianópolis, 2002.

LIDA, I. Ergonomia: projeto e produção. 2. ed. São Paulo: Edgard Blucher, 2005.
LOURENÇO, M.S. et al. Avaliação do perfil ergonômico e nutricional de colaboradores em uma unidade de alimentação e nutrição. In: XIII SIMPEP, 2006; Bauru, São Paulo; 2006.

LOURENÇO, M.S.; MENEZES, L.F. Ergonomia e alimentação coletiva: análise das condições de trabalho em uma Unidade de Alimentação e Nutrição. In: IV CONGRESSO NACIONAL DE EXCELÊNCIA EM GESTÃO. Rio de Janeiro; 2008. Disponível em: $<$ http://www.inovarse.org/filebrowser/downlo ad/8877>. Acesso em: 05 ago. 2016.

MACIEL, G.F.S.V. et al. Aplicação do método de análise ergonômica do trabalho em uma empresa alimentícia de pequeno porte objetivando propostas de melhoria das condições de trabalho. In: XXXIII ENCONTRO NACIONAL DE ENGENHARIA DE PRODUÇÃO, 2013; Salvador, Bahia; 2013.

MATOS, C.H.; PROENÇA, R.P.C.

Condições de trabalho e estado nutricional de operadores do setor de alimentação coletiva: um estudo de caso. Rev. Nutr. v.16, n.4, p.493-502, 2003.

MESQUITA, G.A. et al. Análise da atividade laboral de operadores de caixa de supermercado. In: XII CONGRESSO BRASILEIRO DE ERGONOMIA. Fortaleza, 2004.

OROFINO, C.I. Proposta de educação profissional com base em uma análise ergonômica do trabalho; estudo de caso para as copeiras do hospital universitário da universidade federal de Santa Catarina. Dissertação (Mestrado em Engenharia). Programa de Pós-Graduação em Engenharia de Produção, Universidade Federal de Santa Catarina (UFSC), Florianópolis, 2002.

ROOS, E.C. et al. Food Trucks guiando a inovação: um estudo sobre motivações e inovação no modelo de negócios no setor 
gastronômico de Porto Alegre. In: XVIII SEMINÁRIOS DE ADMINISTRAÇÃO, Universidade Federal do Rio Grande do Sul, 2015, p.1-17.

SÃO PAULO. Secretaria Municipal da Saúde. Portaria SMS-G n ${ }^{\circ} 2619$ de 06 de dezembro de 2011. Regulamento de Boas Práticas e de Controle de condições sanitárias e técnicas das atividades relacionadas à importação, exportação, extração, produção, manipulação, beneficiamento, acondicionamento, transporte, armazenamento, distribuição, embalagem, reembalagem, fracionamento, comercialização e uso de alimentos, águas minerais e de fontes, bebidas, aditivos e embalagens para alimentos. Código Sanitário do Município de São Paulo. Publicada em DOC de 6/12/11, p. 23.

SILVA, D.O. et al. Reconhecimento dos riscos ambientais presentes em unidades de alimentação e nutrição no município de Duque e Caxias, RJ. Saúde Amb. v.2, n.3, p.1-6, 2008. Disponível em:

<http://docplayer.com.br/18503134Reconhecimento-dos-riscos-ambientaispresentes-em-unidades-de-alimentacao-enutricao-no-municipio-de-duque-de-caxiasrj.html>. Acesso em: 05 ago. 2016.

\section{SILVA JÚNIOR, E.A. Manual de Controle} Higiênico-Sanitário em Alimentos. São Paulo: Livraria Valera, 2005.

SIQUEIRA, M.N. et al. Análise ergonômica do trabalho na churrascaria gaúcha. In: XXXI ENCONTRO NACIONAL DE ENGENHARIA DE PRODUÇÃO. Cenário Econômico Mundial Belo Horizonte, MG, Brasil, 04 a 07 de outubro de 2011.

TORRES, M.L. et al. Avaliação do desempenho ergonômico de cozinhas residenciais através da análise comparativa de arranjos físicos. Ambiente Construído, Porto Alegre, v. 6, n. 3, p. 69-90, jul./set. 2006.
TRAEBERT, J. et al. Transição alimentar: problema comum à obesidade e à cárie dentária. Rev. Nutr. v.17, n.2, p. 247-253, 2004.

WHO. World Health Organization. Food safety issues: essential safety requirements for street-vended foods. (Revised edition). 1996. Disponível em: <http://www.who.int/fsf/96-7.pdf>. Acesso em: 15 mar. 2016.

WHO. World Health Organization. Physical status: the use and interpretation of anthropometry. Genebra, p.263-311, 1995. 